\title{
Modeling and Kinematics Simulation of a Mecanum Wheel Platform in RecurDyn
}

\author{
Yunwang Li $\mathbb{D},{ }^{1}$ Sumei Dai $\mathbb{D},{ }^{2}$ Yuwei Zheng, ${ }^{1}$ Feng Tian $\mathbb{D}^{1},{ }^{1}$ and Xucong Yan ${ }^{1}$ \\ ${ }^{1}$ School of Mechatronic Engineering, China University of Mining and Technology, Xuzhou 221116, China \\ ${ }^{2}$ School of Mechanical and Electrical Engineering, Xuzhou Institute of Technology, Xuzhou 221111, China \\ Correspondence should be addressed to Sumei Dai; sumei-dai@hotmail.com
}

Received 30 June 2017; Accepted 18 December 2017; Published 28 January 2018

Academic Editor: Long Cheng

Copyright (c) 2018 Yunwang Li et al. This is an open access article distributed under the Creative Commons Attribution License, which permits unrestricted use, distribution, and reproduction in any medium, provided the original work is properly cited.

\begin{abstract}
The innovative method of modeling and kinematics simulation in RecurDyn are proposed, taking a Mecanum wheel platform(MWP) for omnidirectional wheelchair as research object. In order to study the motion characteristics and mobile performance of the MWP, the virtual prototype simulation model is established in SolidWorks, and virtual prototype simulation is carried out in RecurDyn. The experience of simulation for the MWP in RecurDyn is introduced, and the simulation steps and points for attention are described detailedly. The working states of the mobile system in real environment have been simulated through virtual simulation experiments. Four typical motion models including moving forward, moving laterally, moving laterally in the direction of $45^{\circ}$, and rotation have been simulated in RecurDyn. The simulation results exactly reflect the motion of the MWP. By comparing the simulation results with the theoretical results, there are acceptable errors that are relatively less overall in the simulation results. The simulation results can be used to predict the performance of the platform and evaluate the design rationality, and design quality can be improved according to the exposed problem. This paper can provide reference for the simulation of mobile platform by using RecurDyn.
\end{abstract}

\section{Introduction}

With the development of computer technology and the expansion requirements of engineering application, virtual prototyping technology (VPT) [1-4] has gradually come into people's vision. The virtual prototyping technology, of which the cores are mechanical system kinematics, dynamics, and control theory, is a new engineering technology and has developed rapidly through mature three-dimensional computer graphics technology, graph-based user interface technology, and mature market.

Recursive Dynamic (RecurDyn) [5] is a latest generation of multibody system simulation optimization software developed by FunctionBay company based on recursive algorithm. The relative coordinate system motion equation theory and the complete recursive algorithm are used to solve problems, which make it ideally suitable for solving the multibody dynamics problem of large-scale and complicated contact.

The Mecanum wheel, a kind of omnidirectional wheel, has got a wider range of applications in the field of mobile robots in recent years [6-9]. The movements in any direction on flat surface can be achieved by a combination of three Mecanum wheels or more than three ones. Mecanum wheel platform (MWP) has a high application value in the industrial field. The kinematic characteristic of MWP is the basis of the research on motion control research and application of MWP. The motion simulation of virtual prototyping is an effective research method.

In order to study the motion characteristics and mobile performance of a MWP for an omnidirectional wheelchair, the virtual prototype simulation model was established using SolidWorks software, and virtual prototype simulation was carried out in RecurDyn, before its physical prototype of MWP is being manufactured. The simulation results have promoted the improvement of the prototype design. In this paper, the experience of simulation for the MWP by RecurDyn is introduced, and the simulation steps and points for attention are described detailedly. In the simulation environment of RecurDyn, the simulation analysis of contact collision problem can be carried out quickly and efficiently. 


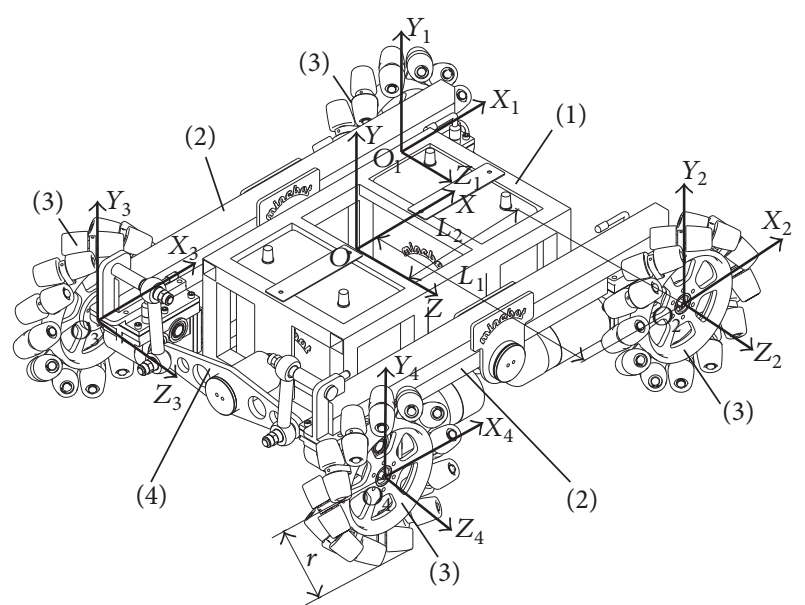

Figure 1: 3D model of the MWP. (1) Main body. (2) Rocker arm. (3) Mecanum wheel. (4) Differential mechanism.

The working state of the mobile system in real environment can be simulated through a variety of virtual simulation experiments. The simulation results can be used to predict the performance of the platform and evaluate the rationality of the design, and design quality can be improved according to the exposed problem.

\section{Virtual Prototype Simulation Model of the Platform}

The 3D modeling function of RecurDyn is relatively weak, so the $3 \mathrm{D}$ virtual prototype model for simulating in RecurDyn is usually developed in other CAD software in order to improve the modeling efficiency. SolidWorks, an interactive CAD/CAE/CAM software, is powerful in the function of design and modeling and widely applied in the design field. In this paper, designing, modeling and assembling of the MWP were completed by SolidWorks. Before being imported into RecurDyn, the model should be simplified to reduce the amount of data processing and improve the efficiency of simulation calculation. Some parts, such as bearings, bolts, and electrical components, that have less impact on the simulation can be ignored, and the main subject required for study must be retained.

2.1. Three-Dimensional Model of Virtual Prototype. The MWP in this paper is mainly composed of four parts: main body, two rocker arms, four Mecanum wheels, and differential mechanism, as shown in Figure 1 [10]. The components such as battery and electrical components are placed in the main body. The rocker arms and differential mechanism are used to combine the main body and the four Mecanum wheels that are driven independently. With the differential mechanism, the platform can adapt to terrain passively, the four wheels can keep contact with the ground simultaneously, and the load of the four wheels is approximately equal. The platform can move omnidirectionally with the Mecanum wheels. In this paper, the virtual prototype simulation model of the MWP is developed by SolidWorks.

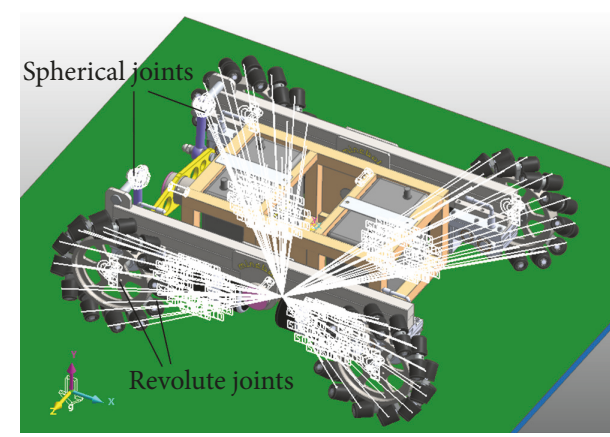

FIGURE 2: Simulation model of the MWP.

2.2. Creating of Joints and Motions. The simplified assembly model can be imported into RecurDyn in Parasolid format. In RecurDyn system, the material and name of the parts and components should be changed, and the Joints between the parts should be defined.

The accuracy of the simulation analysis depends on the accuracy of the above Joints creating. The Joints between the components are created under the premise of confirming the constructional and kinematic relation of the parts. In this simulation model, 103 revolute Joints and 4 spherical Joints are created. 96 of these revolute Joints are between rollers and roller axes of the Mecanum wheel. The 4 spherical Joints are in differential mechanism.

By defining relevant motion functions of the revolute Joints between Mecanum wheels and the rocker arms, the virtual prototype can be enabled to simulate motion according to the predetermined requirements. The STEP functions are used to add motion to system in order to prevent the system simulation abnormity caused by abrupt speed variation in the simulation process. The STEP function, a built-in 3-order polynomial approximation of the step function software, usually used to define a relatively smooth STEP function, is suitable for loading the velocity drive smoothly for the platform.

2.3. Contacts Creating and Parameter Setting. Although the Joints between the parts of the simulation model have been created and the above settings have been completed, the whole model will penetrate the ground under the action of gravity. In order to simulate the motion of the platform, the Contacts between the rollers of the wheels and the ground need to be added. 96 Contacts are generated at one time by using secondary development tool "ProcessNet" to define the above Contacts. This method of Contacts-generating can save operation time and improve modeling efficiency. When the Joints, the Motions, and the Contacts have been defined, the simulation model of MWP has been set up, as shown in Figure 2. According to the Hertz contact theory, the Contact parameters are defined as follows: spring coefficient is $1400 \mathrm{~N} / \mathrm{mm}$, damping coefficient is 10 , dynamic friction coefficient is 0.5 , stiffness exponent is 2.2 , the outer layer material of the roller is set as rubber, and the rest parameters are kept the system default value, as shown in Figure 3. 
TABLE 1: Driving parameters setting of the platform in four typical motion states.

\begin{tabular}{|c|c|c|c|c|}
\hline Motion mode & $\omega_{1} / \mathrm{rad} * \mathrm{~s}^{-1}$ & $\omega_{2} / \mathrm{rad} * \mathrm{~s}^{-1}$ & $\omega_{3} / \mathrm{rad} * \mathrm{~s}^{-1}$ & $\omega_{4} / \mathrm{rad} * \mathrm{~s}^{-1}$ \\
\hline Straight-line motion & $\omega$ & $\omega$ & $\omega$ & $\omega$ \\
\hline Lateral motion & $\omega$ & $-\omega$ & $-\omega$ & $\omega$ \\
\hline Oblique motion at $45^{\circ}$ & 0 & $\omega$ & $\omega$ & 0 \\
\hline Original-place rotation & $\omega$ & $-\omega$ & $\omega$ & $-\omega$ \\
\hline
\end{tabular}

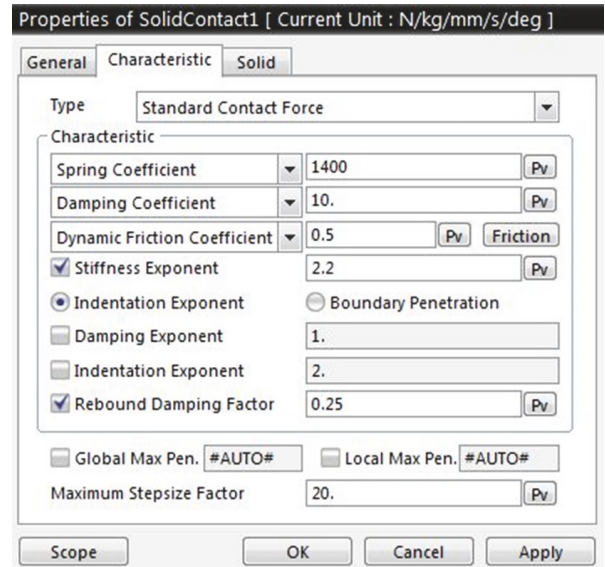

FIgURE 3: Contact parameters setting.

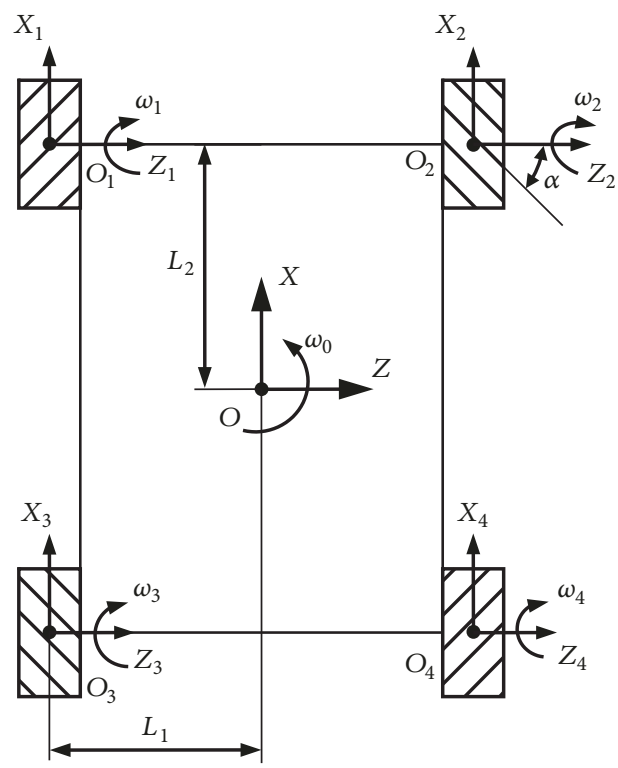

FIgURE 4: Structural schematic diagram of the MWP.

\section{Kinematic Model and Simulation}

3.1. Kinematic Model. The MWP in this paper is a typical four-wheel longitudinal symmetrical layout structure [11], as shown in Figure 4. The coordinate system is set to match the RecurDyn. The bias angles of all the rollers are $\alpha$ and the diagonal lines in Figure 4 indicate the layout of the rollers in contact with the ground. On the assumption that the roller does not skid against the ground and the platform moves on the horizontal plane, the inverse kinematics equation of the system can be established by kinematics analysis [12]:

$$
V_{w}=J(\alpha) \cdot V_{o}
$$

where $V_{w}=\left[\begin{array}{llll}\omega_{1} & \omega_{2} & \omega_{3} & \omega_{4}\end{array}\right]^{\mathrm{T}}$ is the velocity of the wheel. $V_{o}=\left[\begin{array}{lll}v_{x} & v_{z} & \omega_{o}\end{array}\right]^{\mathrm{T}}$ is the generalized velocity of the center point of the platform in the coordinate system XOZ. $J(\alpha)$ is the Jacobi matrix of the inverse equations of motion.

$$
J(\alpha)=\frac{1}{r}\left[\begin{array}{ccc}
1 & \frac{1}{\tan \alpha} & -\frac{L_{1} \tan \alpha+L_{2}}{\tan \alpha} \\
1 & -\frac{1}{\tan \alpha} & \frac{L_{1} \tan \alpha+L_{2}}{\tan \alpha} \\
1 & -\frac{1}{\tan \alpha} & -\frac{L_{1} \tan \alpha+L_{2}}{\tan \alpha} \\
1 & \frac{1}{\tan \alpha} & \frac{L_{1} \tan \alpha+L_{2}}{\tan \alpha}
\end{array}\right] .
$$

The expression of its generalized inverse matrix $J(\alpha)^{+}$is

$$
\begin{aligned}
J(\alpha)^{+} & =\left(J(\alpha)^{T} J(\alpha)\right)^{-1} J(\alpha)^{T}, \\
J(\alpha)^{+} J(\alpha) & =I_{3} .
\end{aligned}
$$

In this model, $\alpha=45^{\circ}$, and the forward kinematics equation of the system is obtained:

$$
\begin{gathered}
{\left[\begin{array}{l}
v_{x} \\
v_{z} \\
\omega_{0}
\end{array}\right]=\frac{r}{4}} \\
\cdot\left[\begin{array}{cccc}
1 & 1 & 1 & 1 \\
1 & -1 & -1 & 1 \\
-\frac{1}{L_{1}+L_{2}} & \frac{1}{L_{1}+L_{2}} & -\frac{1}{L_{1}+L_{2}} & \frac{1}{L_{1}+L_{2}}
\end{array}\right]\left[\begin{array}{l}
\omega_{1} \\
\omega_{2} \\
\omega_{3} \\
\omega_{4}
\end{array}\right] .
\end{gathered}
$$

3.2. Simulation Results and Analysis. According to the inverse kinematics equation of formula (1), the platform moves along the $X$-axis, when $v_{x} \neq 0, v_{z}=0$, and $\omega_{0}=0$, and the rotation speed of each Mecanum wheel is equal to $\omega_{1}=\omega_{2}=\omega_{3}=$ $\omega_{4}=\omega$.

A constant angular velocity drive function is added to the revolute pair between each wheel and the rocker arm: $\omega(t)=$ STEP(TIME, 0, 0, 0.2, PI), then the movement of the platform along $X$-axis can be simulated. Four typical motion states of Mecanum wheel are set to analyze the kinetic characteristics, as shown in Table 1. 
TABLE 2: Comparison between simulation results and theoretical results of the platform motion velocity.

\begin{tabular}{lccc}
\hline Movement type & Simulation results & Theoretical results & Relative error \\
\hline Rectilinear movement $\left|v_{x}\right| / \mathrm{mm}^{-1} \mathrm{~s}^{-1}$ & 438.98 & 439.82 & $0.19 \%$ \\
Lateral movement $\left|v_{\mathrm{z}}\right| / \mathrm{mm} * \mathrm{~s}^{-1}$ & 433.57 & 439.82 & $1.42 \%$ \\
Oblique movement at $45^{\circ}$ & & & 219.91 \\
$\left|v_{x}\right| / \mathrm{mm} * \mathrm{~s}^{-1}$ & 217.41 & 219.91 & $1.14 \%$ \\
$\left|v_{\mathrm{z}}\right| / \mathrm{mm} * \mathrm{~s}^{-1}$ & 217.24 & 0.691 & $1.21 \%$ \\
Original-place rotation $\left|\omega_{0}\right| / \mathrm{rad}^{-1}$ & 0.685 & $0.87 \%$ \\
\hline
\end{tabular}

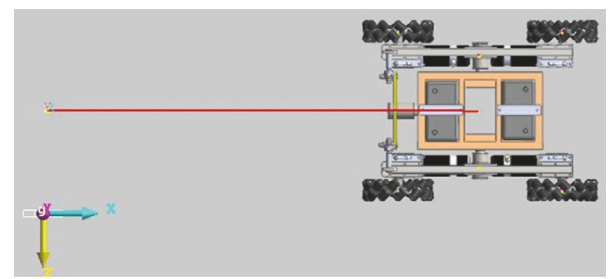

FIGURE 5: Trajectory of the MWP in straight-line motion.

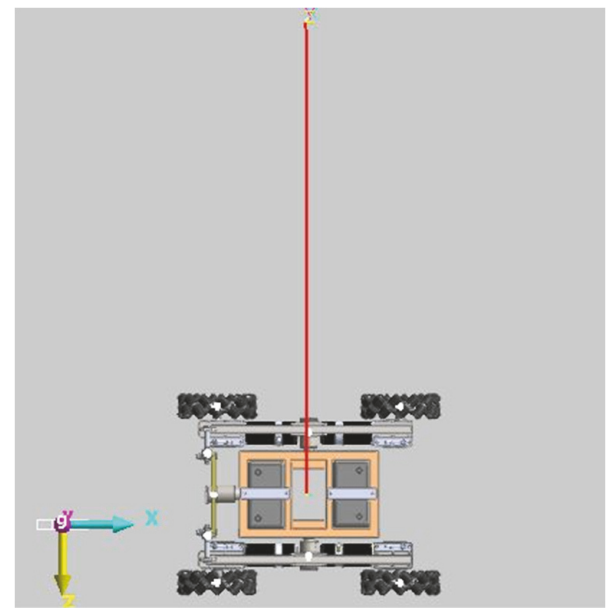

FIgURE 6: Trajectory of the MWP in lateral motion.

In order to better show the motion state of the platform in the simulation process, the trajectory of related Marker Point can be displayed using the Marker Trace command during simulation animation. In the simulation, a Marker is set at the center of the mobile platform. The trajectories of the platform's center in the above four motion modes are shown in Figures 5-8.

Figures 5, 6, and 7 show that the trajectory of the platform's center is a nearly straight line and the orientation of the platform stays the same during its movement. Figure 8 shows the simulation process of the original rotation of the mobile platform. The trajectory of the center of the MWP is a complete circle in the course of the simulation.

The displacement-time curve of the Marker in vertical direction ( $Y$-axis direction) in straight-line motion is shown in Figure 9. It can show that the MWP falls to the ground freely under gravity at first stage of the simulation; the

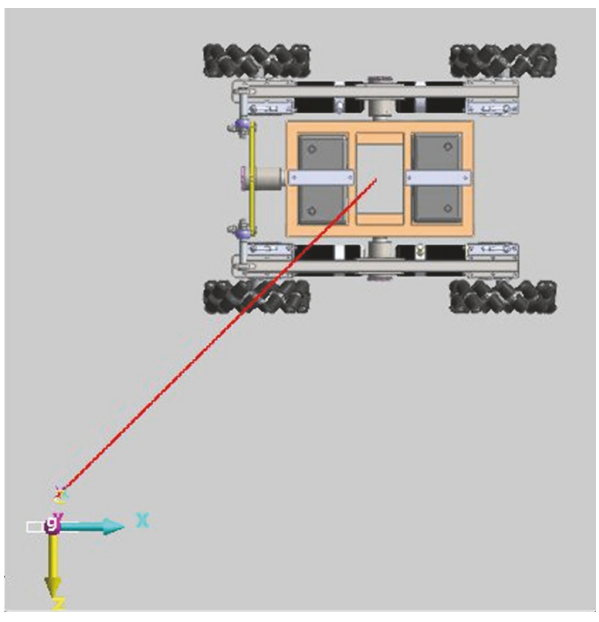

FIgURE 7: Trajectory of the MWP in oblique motion at $45^{\circ}$.

displacement in vertical direction changes rapidly. After the wheels touch the ground, the platform approximately vibrates up and down periodically. The amplitude of vibration is within $1 \mathrm{~mm}$, which can be ignored comparing with the size of the platform. The curve also shows that the Mecanum wheels bring vibration to the mobile platform during the motion.

The velocity-time curves in $X$-axis and $Z$-axis direction in oblique motion at $45^{\circ}$ are drawn according to the simulation results, as shown in Figure 10. From Figure 10, the velocity of the platform increases rapidly from 0 to expected value in the $X, Z$ direction within $0.2 \mathrm{~s}$, consistent with the variation trend of the defined STEP function. Then the velocity values in the $X, Z$ direction fluctuate within a certain range. The velocities in the two directions are basically the same that show the platform move laterally in the direction of $45^{\circ}$ with horizontal line commendably.

According to formula (4), if $r=140 \mathrm{~mm}, L_{1}=313.0 \mathrm{~mm}$, and $L_{2}=323.8 \mathrm{~mm}$, theoretical results of the platform motion velocity can be obtained. The simulation results are compared with the theoretical results in Table 2 . It can be known from Table 2 that there exist varying degrees of errors in simulation results, and the simulation error is relatively less overall. The error is caused by many factors, such as model error, assembly errors, software errors, roller deformation, and skidding. The impact of each factor on the errors still needs to be further studied and is not discussed in detail in this paper. By comparing all simulation results, the rectilinear 


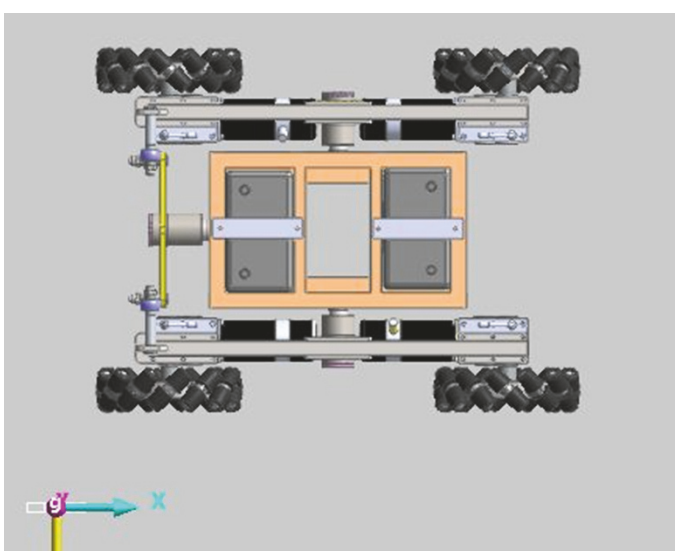

(a)

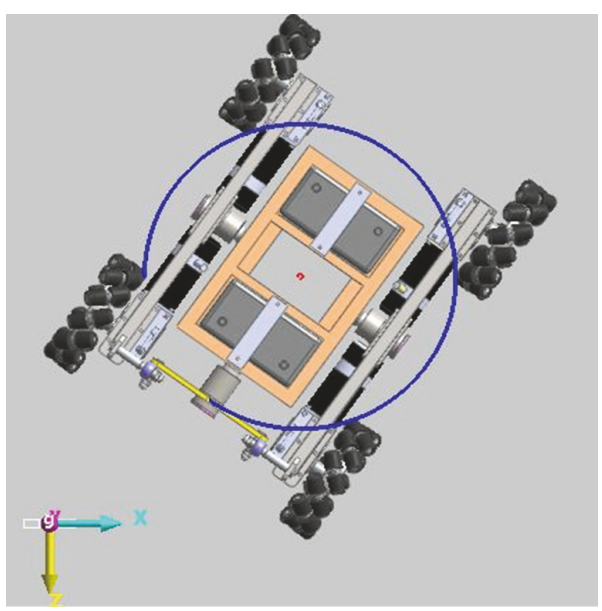

(c)

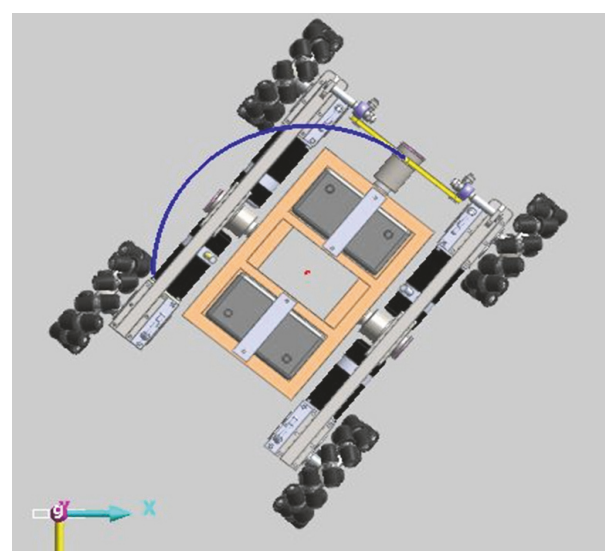

(b)

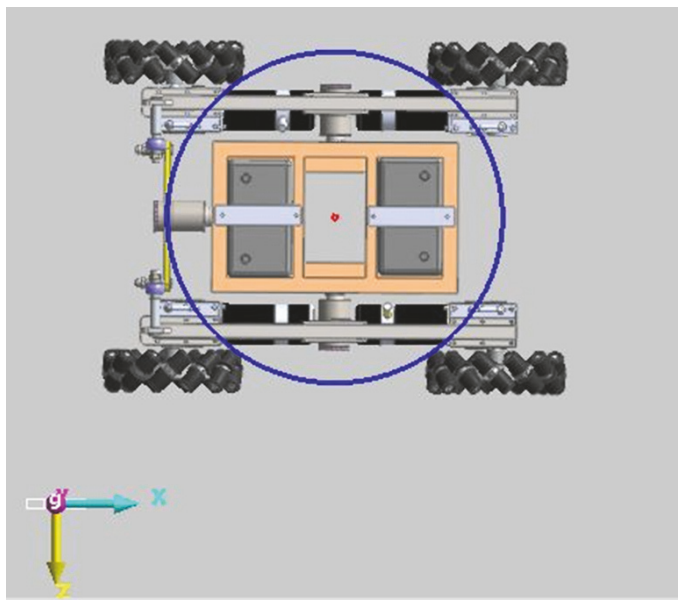

(d)

FIGURE 8: Trajectory of the MWP during the original-place rotation.

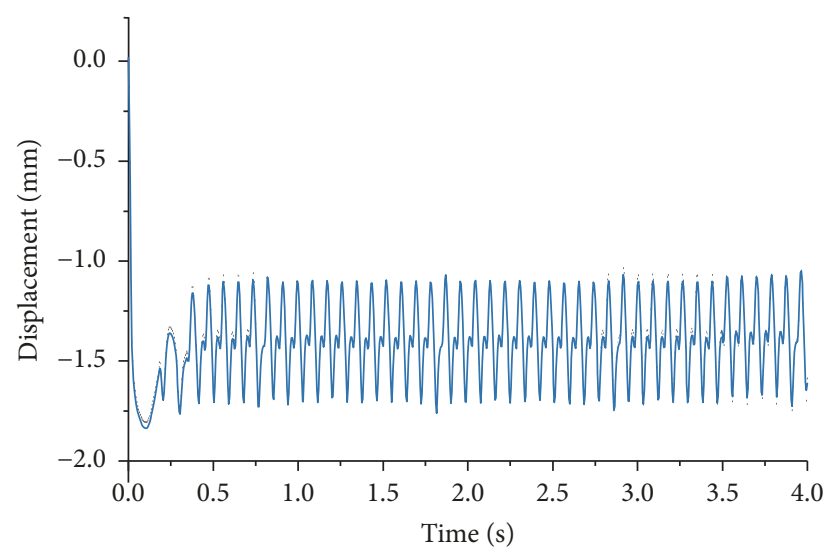

FIGURE 9: Displacement-time curve in the $Y$ direction in straightline motion.

movement's relative error of velocity is minimum, and the error in lateral motion is greater, because the Mecanum wheel is equivalent to the ordinary wheel when the MWP moves forward straightly. The structure of the Mecanum wheel has

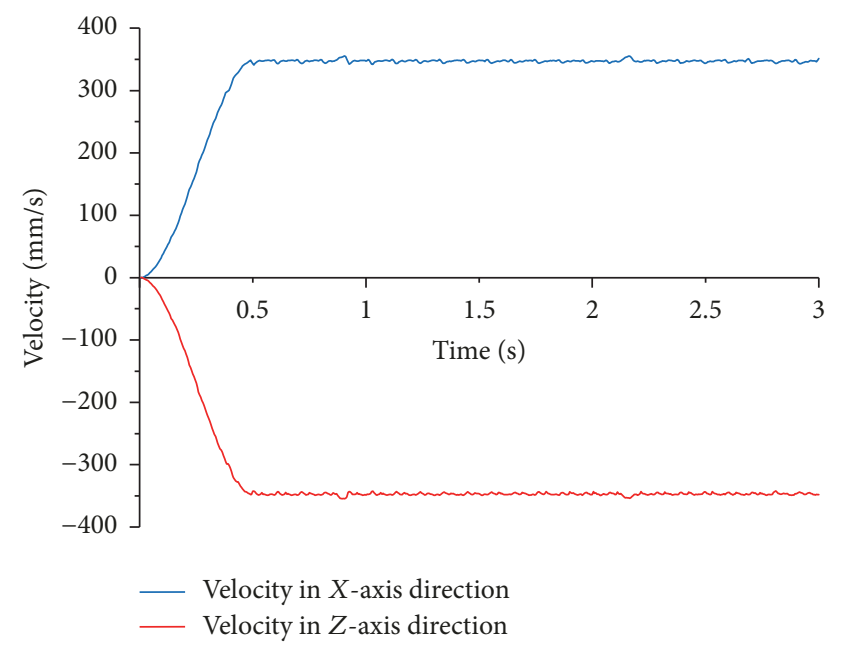

Figure 10: Velocity-time curves in $X$-axis and $Z$-axis direction in oblique motion at $45^{\circ}$.

little influence on the movement of the mobile platform during straight motion but great during lateral motion. 


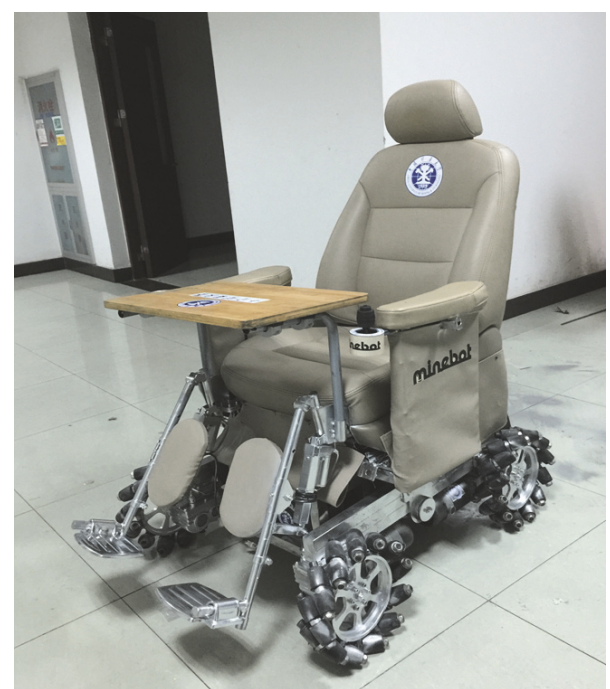

FIGURE 11: The photo of physical prototype of omnidirectional wheelchair.

\section{Conclusion}

In order to study the motion characteristics and mobile performance of a Mecanum wheel platform for an omnidirectional wheelchair, the virtual prototype simulation model is established using SolidWorks, and virtual prototype simulation is carried out based on RecurDyn, before its physical prototype of MWP being manufactured. In this paper, the experience of simulation for the MWP by RecurDyn is introduced, and the simulation steps and points for attention are described detailedly.

The working states of the mobile system in real environment have been simulated through a variety of virtual simulation experiments. Four typical motion modes including moving forward, moving laterally, moving laterally in the direction of $45^{\circ}$ with horizontal line, and rotation have been simulated by RecurDyn. The trajectory of the related Marker that is set at the center of the mobile platform has been displayed using the Marker Trace command during simulation animation. The trajectories in the above four motion modes show the motion state of the platform clearly in the simulation process. The displacement-time curve of the Marker set on the MWP in vertical direction ( $Y$-axis direction) in straight-line motion is drawn. The velocity-time curves of the platform in $X$-axis and $Z$-axis direction in oblique motion at $45^{\circ}$ are drawn according to the simulation results. The trajectories and curves exactly reflect the motion of the MWP. By comparing the simulation results with the theoretical results, there are acceptable errors that are relatively less overall in the simulation results.

The simulation results can be used to predict the performance of the platform and evaluate the design rationality, and design quality can be improved according to the exposed problem. On the basis of RecurDyn simulation, the design of Mecanum wheel mobile platform has been further optimized, and the physical prototype of omnidirectional wheelchair was designed and manufactured, as shown in Figure 11.
This paper shares the experience of modeling and simulation in RecurDyn. The simulation example of the MWP further verifies the feasibility and scalability of the simulation method involved in this paper. This paper can provide reference for the simulation of mobile platform by using RecurDyn.

\section{Conflicts of Interest}

The authors declare that they have no conflicts of interest.

\section{Acknowledgments}

This work was financially supported by the National Natural Science Foundation of China (no. 51675518) and the Priority Academic Program Development of Jiangsu Higher Education Institutions.

\section{References}

[1] G.-L. Xiong, B.-H. Li, and X.-D. Chai, "Virtual prototyping technology," Journal of System Simulation, vol. 13, no. 1, pp. 106109, 2001.

[2] F. Zorriassatine, C. Wykes, R. Parkin, and N. Gindy, "A survey of virtual prototyping techniques for mechanical product development," Proceedings of the Institution of Mechanical Engineers, Part B: Journal of Engineering Manufacture, vol. 217, no. 4, pp. 513-530, 2003.

[3] G. Lu, G. Xue, and Z. Chen, "Design and implementation of virtual interactive scene based on unity 3D," Advanced Materials Research, vol. 317-319, pp. 2162-2167, 2011.

[4] J. Wang, K. Bennett, and E. Guinness, "Virtual Astronaut for Scientific Visualization-A Prototype for Santa Maria Crater on Mars," Future Internet, vol. 4, no. 4, pp. 1049-1068, 2012.

[5] RecurDynTM/Solver Theoretical Manual: 5th Revision. FunctionBay Inc., 2005.

[6] A. Gfrerrer, "Geometry and kinematics of the Mecanum wheel," Computer Aided Geometric Design, vol. 25, no. 9, pp. 784-791, 2008.

[7] L. Lin C and Y. Shih H, "Modeling and adaptive control of an omni-mecanum-wheeled robot," Intelligent Control \& Automation, vol. 2013, no. 2, pp. 166-179, 2013.

[8] A. Shimada, S. Yajima, P. Viboonchaicheep, and K. Samura, "Mecanum-wheel vehicle systems based on position corrective control," in Proceedings of the IECON 2005: 31st Annual Conference of IEEE Industrial Electronics Society, pp. 2077-2082, November 2005.

[9] J. A. Cooney, W. L. Xu, and G. Bright, "Visual dead-reckoning for motion control of a mecanum-wheeled mobile robot," Mechatronics, vol. 14, no. 6, pp. 623-637, 2004.

[10] L. Yunwang, T. Feng, and D. Sumei, "Rocker type omnidirectional mobile platform based on Mecanum wheel," GBCN204250202U.

[11] Y. Wang and D. Chang, "Motion performance analysis and layout selection for motion system with four Mecanum wheels," Journal of Mechanical Engineering, vol. 45, no. 5, pp. 307-316, 2009.

[12] M. O. Tatar, C. Popovici, D. Mandru, I. Ardelean, and A. Plesa, "Design and development of an autonomous omni-directional 
mobile robot with Mecanum wheels," in Proceedings of the 2014 19th IEEE International Conference on Automation, Quality and Testing, Robotics, AQTR 2014, May 2014. 


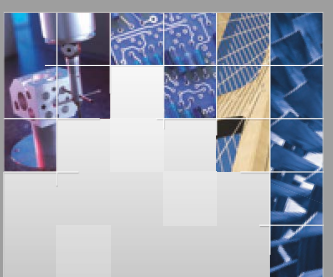

\section{Enfincering}
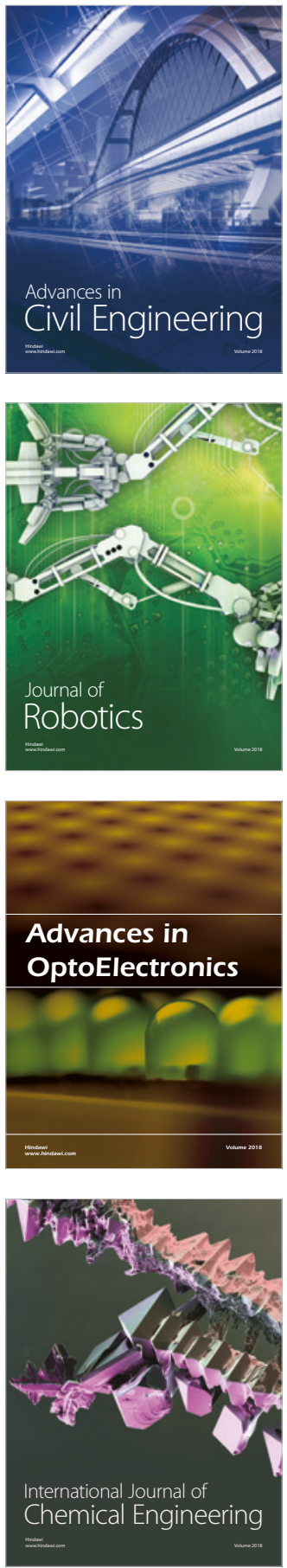

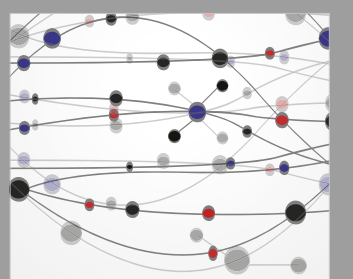

\section{Rotating \\ Machinery}

The Scientific World Journal

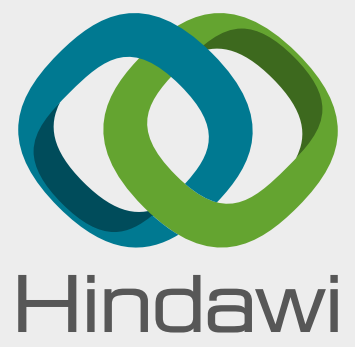

Submit your manuscripts at

www.hindawi.com
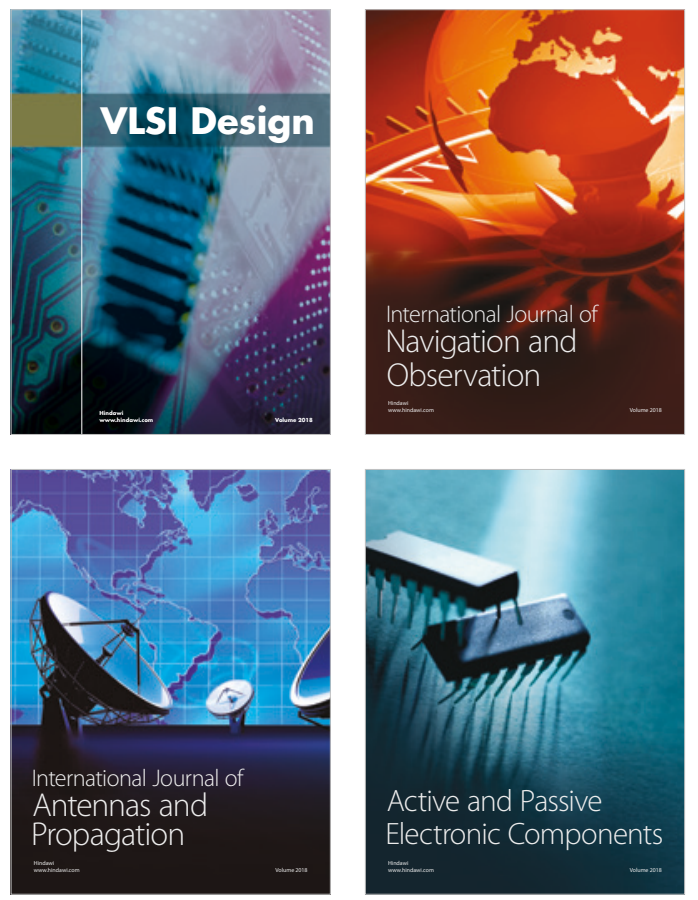
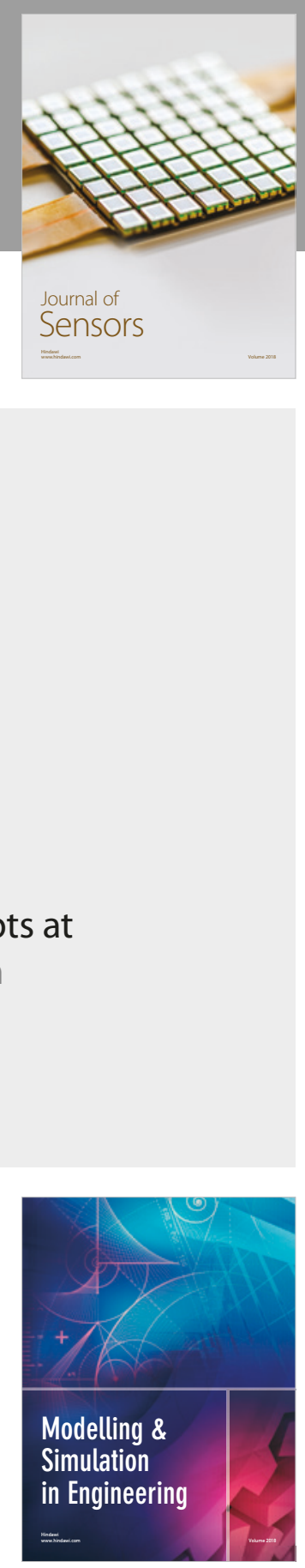

\section{Advances \\ Multimedia}
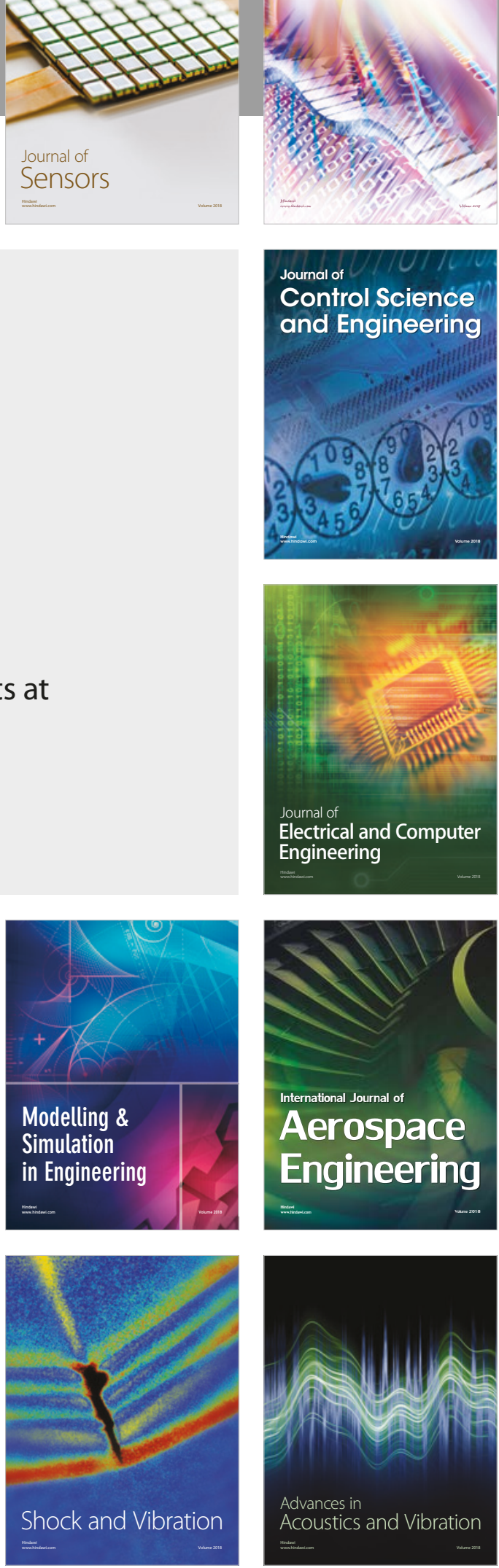